\title{
USES OF 4,5,6,7-TETRAHYDROBENZO[b]THIOPHENE IN THE SYNTHESIS OF PYRIDAZINE, PYRAZOLE, THIAZOLE AND PYRIMIDINE DERIVATIVES TOGETHER WITH THEIR CYTOTOXICITY
}

\author{
Karam A. El-Sharkawy ${ }^{1}$ and Faten I. Hamed ${ }^{2}$ \\ ${ }^{1}$ Faculty of Biotechnology, Chemistry Department, October University of Modern Sciences \\ and Arts (MSA), El-Wahat road, 6 October City, Egypt. \\ ${ }^{2}$ National Organization of Drug Control and Research (NODCAR), P.O. 29, Cairo, A. R. \\ Egypt
}

\begin{abstract}
The reaction of $\mathrm{N}$-phenylbutanamide derivative $\mathbf{1}$ with bromine afforded compound $\mathbf{2}$ which it was directed to reacts with activated methylene groups, malononitrile (3a) and ethylcyanoacetae (3b) to produce compounds $4 \mathbf{a}$ and $\mathbf{4 b}$ respectively, on the other hand the reaction of compound $\mathbf{2}$ with either hydrazine hydrate (8a) or phenylhydrazine $(\mathbf{8 b})$ afforded pyridazine derivatives $\mathbf{1 0 a}, \mathbf{b}$ respectively, Moreover the reaction of compound $\mathbf{2}$ with either potassium cyanide (11a) or potassium thiocyanate (11b) produced compounds 12a,b respectively. Finally the reaction of compound 2 with thiourea (13a) afforded thiazole derivative 14. Compound $4 \mathbf{b}$ reacted with benzenediazonium chloride (5) afforded pyridazine derivative 7. The reactivity of compound 12a was introduced through the reaction with either hydrazine derivatives 8a,b or aromatic aldehydes 16, 18 then compounds $\mathbf{1 5 a}, \mathbf{b}, \mathbf{1 7}, \mathbf{1 9}$ were produced respectively. As extension of compound $\mathbf{1}$ reactions, malononitrile (3a) reacted with compound $\mathbf{1}$ afforded two isomeric compounds $\mathbf{2 0}$ and 21, the latter product $\mathbf{2 0}$ was reacted with either hydrazine derivatives $\mathbf{8 a}, \mathbf{b}$ or thiourea and urea $(\mathbf{1 3 a}, \mathbf{b})$ to produce pyrazole derivatives $\mathbf{2 2} \mathbf{a}, \mathbf{b}$ and pyrimidine derivatives $\mathbf{2 3 a} \mathbf{a}, \mathbf{b}$ respectively. Their cytotoxic activities were tested using three different cell lines.
\end{abstract}

\section{INTRODUCTION}

Thiophene derivatives represent a class of important and well-studied heterocycles (Eicher et al., 2003 and Gronowitz Salo, 1991). The interest in this kind of heterocycles has spread in drug design (Wu et al., 2004). Pyridazine derivatives exhibit an interesting numbers of biological properties such as kinase inhibitors (Kate et al., 2004) and antibacterial agents (Rahul et al., 2006), also pyrazolo-pyridine derivatives it has antibacterial activity (Focks $\boldsymbol{e t}$ al., 2005), on the other hand thiazole derivatives has many biological properties such as antiprotozoal agents (Tapia et al., 2003) and potent antiinflammatory agents (Pawan et al., 1997), finally pyrimidine derivatives has a wide spectrum of biological and pharmacological activities. Thus many synthetic pyrimidines are considered as antiepileptic phenobarbital (Kwan et al., 2004), dihydro-pyrimidinone unit like batzelladine alkaloids have been found to be potent HIV gp120-Human CD4 binding inhibitors (Patil et al., 1995 and Snider et al., 1996).

In this article we have synthesized some new heterocyclic compounds containing tetrahydrobenzo $[b]$ thiophene moiety to try to improve their biological evaluations as well as their cytotoxic activity. 


\section{EXPERMENTAL}

\section{Synthetic methods, analytical and spectral data}

All melting points were determined in open capillaries and are uncorrected. Elemental analyses were performed on a Yanaco CHNS Corder elemental analyzer (Japan). IR spectra were measured using $\mathrm{KBr}$ discs on a Pye Unicam SP-1000 spectrophotometer. ${ }^{1} \mathrm{H}$ NMR spectra were measured on a Varian EM 390-200 $\mathrm{MHz}$ instrument in $\mathrm{CD}_{3} \mathrm{SOCD}_{3}$ as solvent using TMS as internal standard and chemical shifts are expressed as $\delta \mathrm{ppm}$. Mass spectra were recorded on Kratos (75 eV) MS equipment (Germany).

\section{4-Bromo-2-[(3-cyano-4,5,6,7-tetrahydro-benzo[b]thiophen-2-yl)-hydrazono]-3-oxo-N- phenyl-butanamide (2)}

To a solution of compound $1(3.66 \mathrm{~g}, 0.01 \mathrm{~mol})$ in glacial acetic acid $(40 \mathrm{~mL})$ at $60{ }^{\circ} \mathrm{C}$, bromine $(0.50 \mathrm{ml})$ in acetic acid solution $(10 \mathrm{~mL})$ was added drop wise. The reaction mixture, after addition of all bromine solution, was kept at room temperature for $1 \mathrm{~h}$ with continuous stirring. The solid product, formed upon pouring onto ice/water was collected by filtration.

Compound 2: Pale brown crystals from ethanol, yield: $88 \%(3.920 \mathrm{~g}) ; \mathrm{mp}: 124^{\circ} \mathrm{C}$. IR (KBr): $\mathrm{v} / \mathrm{cm}^{-1}=3479-3331(2 \mathrm{NH}), 3053\left(\mathrm{CH}\right.$-aromatic), $2888\left(\mathrm{CH}_{2}\right), 2225(\mathrm{CN}), 1705,1689(2$ $\mathrm{CO}), 1633(\mathrm{C}=\mathrm{C}) .{ }^{1} \mathrm{H}$ NMR $\left(\mathrm{DMSO}_{6}\right): \delta=1.74-1.79\left(\mathrm{~m}, 4 \mathrm{H}, 2 \mathrm{CH}_{2}\right), 2.11-2.18(\mathrm{~m}, 4 \mathrm{H}$, $\left.2 \mathrm{CH}_{2}\right), 3.88\left(\mathrm{~s}, 2 \mathrm{H}, \mathrm{CH}_{2}\right), 7.27-7.38\left(\mathrm{~m}, 5 \mathrm{H}, \mathrm{C}_{6} \mathrm{H}_{5}\right), 8.32,9.44\left(2 \mathrm{~s}, 2 \mathrm{H}, \mathrm{D}_{2} \mathrm{O}\right.$-exchangeable, 2NH). MS (relative intensity) $\mathrm{m} / \mathrm{z}$ : 446, 444. Analysis for $\mathrm{C}_{19} \mathrm{H}_{17} \mathrm{BrN}_{4} \mathrm{O}_{2} \mathrm{~S}$ Calcd: C, 51.24; H, 3.85; N, 12.58; S, 7.20. Found: C, 51.48 H, 4.02; N, 12.39; S, $7.48 \%$.

2-(3-Cyano-2-hydrazinyl-4,5,6,7-tetrahydrobenzo[b]thiophene)-3(bromomethyl) $\quad$-4,4dicyano-N-phenylbut-3-enamide (4a) and Ethyl 4-(phenylcarbamoyl)-4-(3-cyano-2hydrazinyl-4,5,6,7-tetrahydrobenzo $[b]$-thiophene)-3-(bromomethyl)-2-cyanobut-2enoate (4b)

To a solution of compound $2(2.22 \mathrm{~g}, 0.005 \mathrm{~mol})$ in 1,4-dioxane $(40 \mathrm{~mL})$ containing triethylamine $(0.50 \mathrm{~mL})$, either malononitrile $(3 \mathbf{a}, 0.33 \mathrm{~g}, 0.005 \mathrm{~mol})$ or ethyl cyanoacetate $(3 \mathbf{b}, 0.57 \mathrm{~g}, 0.005 \mathrm{~mol})$ was added. The reaction mixture was heated under reflux for $3 \mathrm{~h}$ then left to cool and the formed solid product, so formed was collected by filtration.

Compound 4a: Yellow crystals from ethanol, yield: $82 \%(2.03 \mathrm{~g})$; mp: $288^{\circ} \mathrm{C}$. IR (KBr): $\mathrm{v} / \mathrm{cm}^{-1}=3465-3328(2 \mathrm{NH}), 3056(\mathrm{CH}$-aromatic $), 2885\left(\mathrm{CH}_{2}\right), 2227-2220(3 \mathrm{CN}), 1687$ $(\mathrm{CO}), 1633(\mathrm{C}=\mathrm{C}) .{ }^{1} \mathrm{H}$ NMR $\left(\mathrm{DMSO}_{-} \mathrm{d}_{6}\right): \delta=1.71-1.76\left(\mathrm{~m}, 4 \mathrm{H}, 2 \mathrm{CH}_{2}\right), 2.15-2.19(\mathrm{~m}, 4 \mathrm{H}$, $\left.2 \mathrm{CH}_{2}\right), 3.93\left(\mathrm{~s}, 2 \mathrm{H}, \mathrm{CH}_{2}\right), 7.28-7.39\left(\mathrm{~m}, 5 \mathrm{H}, \mathrm{C}_{6} \mathrm{H}_{5}\right), 8.39,9.42\left(2 \mathrm{~s}, 2 \mathrm{H}, \mathrm{D}_{2} \mathrm{O}\right.$-exchangeable, 2NH). MS (relative intensity) m/z: 494, 492. Analysis for $\mathrm{C}_{22} \mathrm{H}_{17} \mathrm{BrN}_{6} \mathrm{OS}$ Calcd: C, 53.56; H, 3.47; N, 17.03; S, 6.50. Found: C, 53.72; H, 3.55; N, 16.82; S, $6.36 \%$.

Compound 4b: Yellow crystals from ethanol, yield: $77 \%(2.08 \mathrm{~g})$; mp: $193{ }^{\circ} \mathrm{C}$. IR $(\mathrm{KBr})$ : $\mathrm{v} / \mathrm{cm}^{-1}=3471-3347(2 \mathrm{NH}), 3058(\mathrm{CH}$-aromatic $), 2882\left(\mathrm{CH}_{2}\right), 2227,2222(\mathrm{CN}), 1785,1689$ (2CO), $1634(\mathrm{C}=\mathrm{C}) .{ }^{1} \mathrm{H}$ NMR $\left(\mathrm{DMSO}_{-} \mathrm{d}_{6}\right): \delta=1.13\left(\mathrm{t}, 3 \mathrm{H}, \mathrm{J}=7.55 \mathrm{~Hz}, \mathrm{CH}_{3}\right), 1.70-1.77(\mathrm{~m}$, $\left.4 \mathrm{H}, 2 \mathrm{CH}_{2}\right), 1.97-2.05\left(\mathrm{~m}, 4 \mathrm{H}, 2 \mathrm{CH}_{2}\right), 3.81\left(\mathrm{~s}, 2 \mathrm{H}, \mathrm{CH}_{2}\right), 4.22\left(\mathrm{q}, 2 \mathrm{H}, \mathrm{J}=7.55 \mathrm{~Hz}, \mathrm{CH}_{2}\right)$, 7.29-7.41 (m, 5H, $\left.\mathrm{C}_{6} \mathrm{H}_{5}\right), 8.33,9.40\left(2 \mathrm{~s}, 2 \mathrm{H}, \mathrm{D}_{2} \mathrm{O}\right.$-exchangeable, 2NH). MS (relative intensity) $\mathrm{m} / \mathrm{z}$ : 541, 539. Analysis for $\mathrm{C}_{24} \mathrm{H}_{22} \mathrm{BrN}_{5} \mathrm{O}_{3} \mathrm{~S}$ Calcd: $\mathrm{C}, 53.34 ; \mathrm{H}, 4.10 ; \mathrm{N}, 12.96$; $\mathrm{S}$, 5.93. Found: C, 53.51; H, 4.27; N, $13.07 \mathrm{~S}, 6.22 \%$.

\section{2-(3-Cyano-2-hydrazinyl-4,5,6,7-tetrahydrobenzo[b]thiophene)-2-(3-bromo-5-cyano- 1,6-dihydro-6-oxo-1-phenylpyridazin-4-yl)-N-phenylacetamide (7)}

To a solution of compound $\mathbf{4 b}(2.7 \mathrm{~g}, 0.005 \mathrm{~mol})$ in ethanol $(40 \mathrm{~mL})$ containing sodium hydroxide $(10 \mathrm{~mL}, 10 \%)$, a cold solution of benzenediazonium chloride (5) [prepared by the addition of sodium nitrite $(0.35 \mathrm{~g}, 0.005 \mathrm{~mol})$ solution (in $10 \mathrm{~mL}$ water) to a cold solution of aniline $(0.47 \mathrm{~g}, 0.005 \mathrm{~mol})$ in concentrated acetic/hydrochloric acid (10:3) with continuous stirring] was added with continuous stirring. The reaction mixture was stirred for an addition $1 \mathrm{~h}$ at room temperature and the formed solid product was collected by filtration. 
Compound 7: Pale yellow crystals from 1,4 dioxane, yield: $64 \%(1.92 \mathrm{~g})$; mp: $170-172{ }^{\circ} \mathrm{C}$. IR (KBr): v/cm ${ }^{-1}=3477-3320(2 \mathrm{NH}), 3054\left(\mathrm{CH}\right.$-aromatic), $2880\left(\mathrm{CH}_{2}\right), 2224,2221(2 \mathrm{CN})$, 1688, $1684(2 \mathrm{CO}), 1636(\mathrm{C}=\mathrm{C}) .{ }^{1} \mathrm{H}$ NMR $\left(\mathrm{DMSO}-\mathrm{d}_{6}\right): \delta=1.73-1.79\left(\mathrm{~m}, 4 \mathrm{H}, 2 \mathrm{CH}_{2}\right), 1.89-$ $1.99\left(\mathrm{~m}, 4 \mathrm{H}, 2 \mathrm{CH}_{2}\right), 7.24-7.43\left(\mathrm{~m}, 10 \mathrm{H}, 2 \mathrm{C}_{6} \mathrm{H}_{5}\right), 8.32,9.40\left(2 \mathrm{~s}, 2 \mathrm{H}, \mathrm{D}_{2} \mathrm{O}\right.$-exchangeable, 2NH). MS (relative intensity) m/z: 599, 597. Analysis for $\mathrm{C}_{28} \mathrm{H}_{20} \mathrm{BrN}_{7} \mathrm{O}_{2} \mathrm{~S}$ Calcd: C, 56.19; H, 3.37; N, 16.38; S, 5.36. Found: C, 56.05; H, 3.66; N, 16.52; S, $5.57 \%$.

\section{3-Cyano-2-azo-(6-phenylamino-4-hydroxypyridazine-5-yl)-4,5,6,7-tetrahydro- benzo[b]thiophene (10a) and 3-Cyano-2-azo-(6-phenylamino-1-phenyl-4-hydroxy - pyridazine-5-yl)-4,5,6,7-tetrahydrobenzo- $[b]$ thiophene $(10 b)$}

To a solution of compound $2(2.22 \mathrm{~g}, 0.005 \mathrm{~mol})$ in ethanol $(40 \mathrm{~mL})$, either hydrazine hydrate $(\mathbf{8 a}, 0.30 \mathrm{~mL}, 0.005 \mathrm{~mol})$ or phenylhydrazine $(\mathbf{8 b}, 0.60 \mathrm{~g}, 0.005 \mathrm{~mol})$ was added. The reaction mixture in each case was heated under reflux for $4 \mathrm{~h}$ then left to cool. The solid product, formed upon pouring onto ice/water containing few drops of hydrochloric acid (till pH 6) was collected by filtration.

Compound 10a: Yellow crystals from ethanol, yield: $68 \%(1.28 \mathrm{~g})$; mp: $200-202{ }^{\circ} \mathrm{C}$. IR $(\mathrm{KBr}): \mathrm{v} / \mathrm{cm}^{-1}=3522-3348(\mathrm{OH}, \mathrm{NH}), 3053(\mathrm{CH}$-aromatic $), 2886\left(\mathrm{CH}_{2}\right), 2224(\mathrm{CN}), 1638$ $(\mathrm{C}=\mathrm{C}) .{ }^{1} \mathrm{H}$ NMR $\left(\mathrm{DMSO}_{-} \mathrm{d}_{6}\right): \delta=1.73-1.78\left(\mathrm{~m}, 4 \mathrm{H}, 2 \mathrm{CH}_{2}\right), 2.21-2.26\left(\mathrm{~m}, 4 \mathrm{H}, 2 \mathrm{CH}_{2}\right), 6.83$ (s, $1 \mathrm{H}$, pyridazine $\mathrm{H}-3), 7.29-7.42\left(\mathrm{~m}, 5 \mathrm{H}, \mathrm{C}_{6} \mathrm{H}_{5}\right), 8.28\left(\mathrm{~s}, 1 \mathrm{H}, \mathrm{D}_{2} \mathrm{O}\right.$-exchangeable, $\left.\mathrm{NH}\right), 9.38$ (s, $1 \mathrm{H}, \mathrm{D}_{2} \mathrm{O}$-exchangeable, $\mathrm{OH}$ ). MS (relative intensity) $\mathrm{m} / \mathrm{z}$ : 376 . Analysis for $\mathrm{C}_{19} \mathrm{H}_{16} \mathrm{~N}_{6} \mathrm{OS}$ Calcd: C, 60.62; H, 4.28; N, 23.33; S, 8.52. Found: C, 60.93; H, 4.09; N, 23.59; S, 8.35\%.

Compound 10b: Yellow crystals from ethanol, yield: $81 \%(1.84 \mathrm{~g}) ; \mathrm{mp}: 162-163{ }^{\circ} \mathrm{C}$. IR (KBr): v/cm ${ }^{-1}=3555-3337(\mathrm{OH}, 2 \mathrm{NH}), 3056\left(\mathrm{CH}\right.$-aromatic), $2898\left(\mathrm{CH}_{2}\right), 2223(\mathrm{CN}), 1632$ $(\mathrm{C}=\mathrm{C}) .{ }^{1} \mathrm{H}$ NMR $\left(\mathrm{DMSO}_{-} \mathrm{d}_{6}\right): \delta=1.64-1.72\left(\mathrm{~m}, 4 \mathrm{H}, 2 \mathrm{CH}_{2}\right), 2.06-2.14\left(\mathrm{~m}, 4 \mathrm{H}, 2 \mathrm{CH}_{2}\right), 6.94$ (s, $1 \mathrm{H}$, pyridazine $\mathrm{H}-3), 7.28-7.42\left(\mathrm{~m}, 10 \mathrm{H}, 2 \mathrm{C}_{6} \mathrm{H}_{5}\right), 8.32,8.53\left(2 \mathrm{~s}, 2 \mathrm{H}, \mathrm{D}_{2} \mathrm{O}\right.$-exchangeable, $2 \mathrm{NH}), 9.18\left(\mathrm{~s}, 1 \mathrm{H}, \mathrm{D}_{2} \mathrm{O}\right.$-exchangeable, $\left.\mathrm{OH}\right)$. MS (relative intensity) m/z: 454. Analysis for $\mathrm{C}_{25} \mathrm{H}_{22} \mathrm{~N}_{6} \mathrm{OS}$ Calcd: C, 66.06; H, 4.88; N, 18.49; S, 7.05. Found: C, 65.81; H, 4.92; N, $18.57 ; \mathrm{S}, 7.32 \%$.

\section{2-(3-Cyano-2-hydrazinyl-4,5,6,7-tetrahydrobenzo[b]thiophene)-4-cyano-3-oxo-N- phenylbutanamide (12a) and 2-(3-Cyano-2-hydrazinyl-4,5,6,7-tetrahydrobenzo [b] thiophene)- 3-oxo-N-phenyl-4-thiocyanatobutanamide (12b)}

The solution of compound $2(4.44 \mathrm{~g}, 0.01 \mathrm{~mol})$ in ethanol $(50 \mathrm{~mL})$ either potassium cyanide (11a, $1.30 \mathrm{~g}, 0.02 \mathrm{~mol})$ or potassium thiocyanate $(\mathbf{1 1 b}, 1.94 \mathrm{~g}, 0.02 \mathrm{~mol})$ solution in water $(10 \mathrm{~mL})$ was added drop wise and the reaction mixture was heated in water bath at $60{ }^{\circ} \mathrm{C}$ for 1h. The whole reaction mixture was stirred at room temperature for an additional $3 \mathrm{~h}$ then poured onto ice/water and few drops of hydrochloric acid were added, the formed solid product was collected by filtration.

Compound 12a: White crystals from ethanol, yield: $58 \%(2.270 \mathrm{~g}) ; \mathrm{mp}: 178-179{ }^{\circ} \mathrm{C}$. IR $(\mathrm{KBr}): \mathrm{v} / \mathrm{cm}^{-1}=3444-3328(2 \mathrm{NH}), 3055\left(\mathrm{CH}\right.$-aromatic), $2918\left(\mathrm{CH}_{2}\right), 2226,2220(2 \mathrm{CN})$, 1688, 1685 (2 CO), $1633(\mathrm{C}=\mathrm{C}) .{ }^{1} \mathrm{H}$ NMR $\left(\mathrm{DMSO}-\mathrm{d}_{6}\right): \delta=1.71-1.75\left(\mathrm{~m}, 4 \mathrm{H}, 2 \mathrm{CH}_{2}\right), 2.19$ $2.27\left(\mathrm{~m}, 4 \mathrm{H}, 2 \mathrm{CH}_{2}\right), 3.93\left(\mathrm{~s}, 2 \mathrm{H}, \mathrm{CH}_{2}\right), 7.27-7.36\left(\mathrm{~m}, 5 \mathrm{H}, \mathrm{C}_{6} \mathrm{H}_{5}\right), 8.26,8.45\left(2 \mathrm{~s}, 2 \mathrm{H}, \mathrm{D}_{2} \mathrm{O}-\right.$ exchangeable, $2 \mathrm{NH}$ ). MS (relative intensity) $\mathrm{m} / \mathrm{z}$ : 391. Analysis for $\mathrm{C}_{20} \mathrm{H}_{17} \mathrm{~N}_{5} \mathrm{O}_{2} \mathrm{~S}$ Calcd: $\mathrm{C}$, 61.37; H, 4.38; N, 17.89; S, 8.19. Found: C, 61.58; H, 4.59; N, 17.92; S, 8.28\%.

Compound 12b: Yellow crystals from ethanol, yield: $74 \%(3.133 \mathrm{~g})$; mp: $184-186{ }^{\circ} \mathrm{C}$. IR $(\mathrm{KBr}): \mathrm{v} / \mathrm{cm}^{-1}=3476-3332(2 \mathrm{NH}), 3058\left(\mathrm{CH}\right.$-aromatic), $2916\left(\mathrm{CH}_{2}\right), 2222,2220(2 \mathrm{CN})$, $1636(\mathrm{C}=\mathrm{C}) .{ }^{1} \mathrm{H}$ NMR $\left(\mathrm{DMSO}-\mathrm{d}_{6}\right): \delta=1.62-1.70\left(\mathrm{~m}, 4 \mathrm{H}, 2 \mathrm{CH}_{2}\right), 1.98-2.09\left(\mathrm{~m}, 4 \mathrm{H}, 2 \mathrm{CH}_{2}\right)$, $3.89\left(\mathrm{~s}, 2 \mathrm{H}, \mathrm{CH}_{2}\right), 7.28-7.42\left(\mathrm{~m}, 5 \mathrm{H}, \mathrm{C}_{6} \mathrm{H}_{5}\right), 8.34,8.58\left(2 \mathrm{~s}, 2 \mathrm{H}, \mathrm{D}_{2} \mathrm{O}\right.$-exchangeable, $\left.2 \mathrm{NH}\right)$. 
MS (relative intensity) m/z: 423. Analysis for $\mathrm{C}_{20} \mathrm{H}_{17} \mathrm{~N}_{5} \mathrm{O}_{2} \mathrm{~S}_{2}$ Calcd: $\mathrm{C}, 56.72 ; \mathrm{H}, 4.05 ; \mathrm{N}$, 16.54; S, 15.14. Found: C, 56.82; H, 3.89; N, 16.83; S, $15.08 \%$.

\section{2-(3-Cyano-2-hydrazinyl-4,5,6,7-tetrahydrobenzo[b]thiophene)-2-(2-amino-thiazol-4- yl)-N-phenylacetamide (14)}

To a solution of compound $2(4.44 \mathrm{~g}, 0.01 \mathrm{~mol})$ in ethanol $(50 \mathrm{~mL})$, thiourea $(\mathbf{1 3 a}, 0.76 \mathrm{~g}$, $0.01 \mathrm{~mol}$ ) was added. The reaction mixture was heated under reflux for $3 \mathrm{~h}$ then left to cool and the formed solid product upon pouring onto ice/water containing few drops of sodium hydroxide $(5 \%)$ was collected by filtration.

Compound 14: Yellow crystals from DMF, yield: $65 \%(2.746 \mathrm{~g})$; mp: 252-253 ${ }^{\circ} \mathrm{C}$. IR $(\mathrm{KBr}): \mathrm{v} / \mathrm{cm}^{-1}=3483-3343\left(\mathrm{NH}_{2}, 2 \mathrm{NH}\right), 3057\left(\mathrm{CH}\right.$-aromatic), $2883\left(\mathrm{CH}_{2}\right), 2224(\mathrm{CN}), 1687$ $(\mathrm{CO}), 1638(\mathrm{C}=\mathrm{C}) .{ }^{1} \mathrm{H}$ NMR $\left(\mathrm{DMSO}_{-} \mathrm{d}_{6}\right): \delta=1.67-1.74\left(\mathrm{~m}, 4 \mathrm{H}, 2 \mathrm{CH}_{2}\right), 2.02-2.08(\mathrm{~m}, 4 \mathrm{H}$, $\left.2 \mathrm{CH}_{2}\right), 4.22$ (s, 2H, $\mathrm{D}_{2} \mathrm{O}$ exchangeable, $\left.\mathrm{NH}_{2}\right), 6.83$ (s, $1 \mathrm{H}$, thiazole $\left.\mathrm{H}-5\right), 7.29-7.43(\mathrm{~m}, 5 \mathrm{H}$, $\left.\mathrm{C}_{6} \mathrm{H}_{5}\right), 8.24,8.83\left(2 \mathrm{~s}, 2 \mathrm{H}, \mathrm{D}_{2} \mathrm{O}\right.$-exchangeable, 2NH). MS (relative intensity) m/z: 422. Analysis for $\mathrm{C}_{20} \mathrm{H}_{18} \mathrm{~N}_{6} \mathrm{OS}_{2}$ Calcd: C, 56.85; H, 4.29; N, 19.89; S, 15.18. Found: C, 56.59; H, $4.42 ; \mathrm{N}, 19.58 ; \mathrm{S}, 15.42 \%$.

\section{2-(3-Cyano-2-hydrazinyl-4,5,6,7-tetrahydrobenzo[b]thiophene)-2-(3-amino-1H-pyrazol- 5-yl)-N-phenylacetamide (15a) and 2-(3-Cyano-2-hydrazinyl-4,5,6,7- tetrahydrobenzo $[b]$ thiophene)-2-(3-amino-1-phenyl-1 $H$-pyrazol-5-yl)-N-phenyl- acetamide (15b)}

To a solution of compound $12 \mathrm{a}(3.91 \mathrm{~g}, 0.01 \mathrm{~mol})$ in ethanol $(40 \mathrm{~mL})$ either hydrazine hydrate $(\mathbf{8 a}, 0.50 \mathrm{~g}, 0.01 \mathrm{~mol})$ or phenyl hydrazine $(\mathbf{8 b}, 1.08 \mathrm{~g}, 0.01 \mathrm{~mol})$ was added. The reaction mixture was heated under reflux for $4 \mathrm{~h}$ then poured onto ice/water containing few drops of hydrochloric acid and the formed solid product was collected by filtration.

Compound 15a: Yellow crystals from ethanol, yield: $76 \%(3.081 \mathrm{~g})$; mp: $285-287{ }^{\circ} \mathrm{C}$. IR (KBr): v/cm $\mathrm{cm}^{-1}=3485-3321\left(\mathrm{NH}_{2}, 3 \mathrm{NH}\right), 3058\left(\mathrm{CH}\right.$-aromatic), $2884\left(\mathrm{CH}_{2}\right), 2224(\mathrm{CN}), 1689$ (CO), $1636(\mathrm{C}=\mathrm{C}) .{ }^{1} \mathrm{H}$ NMR (DMSO-d $\left.{ }_{6}\right): \delta=1.70-1.77\left(\mathrm{~m}, 4 \mathrm{H}, 2 \mathrm{CH}_{2}\right), 2.19-2.28(\mathrm{~m}, 4 \mathrm{H}$, $\left.2 \mathrm{CH}_{2}\right), 4.66\left(\mathrm{~s}, 2 \mathrm{H}, \mathrm{D}_{2} \mathrm{O}\right.$ exchangeable, $\left.\mathrm{NH}_{2}\right), 6.82(\mathrm{~s}, 1 \mathrm{H}$, pyrazole $\mathrm{H}-4), 7.29-7.43(\mathrm{~m}, 5 \mathrm{H}$, $\left.\mathrm{C}_{6} \mathrm{H}_{5}\right), 8.34,8.46,9.11\left(3 \mathrm{~s}, 3 \mathrm{H}, \mathrm{D}_{2} \mathrm{O}\right.$-exchangeable, $\left.3 \mathrm{NH}\right)$. MS (relative intensity) m/z: 405. Analysis for $\mathrm{C}_{20} \mathrm{H}_{19} \mathrm{~N}_{7} \mathrm{OS}$ Calcd: C, 59.24; H, 4.72; N, 24.18; S, 7.91. Found: C, 58.98; H, $4.61 ; \mathrm{N}, 24.42 ; \mathrm{S}, 8.20 \%$.

Compound 15b: Yellow crystals from ethanol, yield: $73 \%(3.515 \mathrm{~g})$; mp: $189-191{ }^{\circ} \mathrm{C}$. IR (KBr): v/cm $\mathrm{cm}^{-1}=3475-3331\left(\mathrm{NH}_{2}, 2 \mathrm{NH}\right), 3054\left(\mathrm{CH}\right.$-aromatic), $2897\left(\mathrm{CH}_{2}\right), 2221(\mathrm{CN}), 1687$ (CO), $1638(\mathrm{C}=\mathrm{C}) .{ }^{1} \mathrm{H}$ NMR $\left(\mathrm{DMSO}_{-} \mathrm{d}_{6}\right): \delta=1.72-1.77\left(\mathrm{~m}, 4 \mathrm{H}, 2 \mathrm{CH}_{2}\right), 2.15-2.22(\mathrm{~m}, 4 \mathrm{H}$, $\left.2 \mathrm{CH}_{2}\right), 4.68\left(\mathrm{~s}, 2 \mathrm{H}, \mathrm{D}_{2} \mathrm{O}\right.$ exchangeable, $\left.\mathrm{NH}_{2}\right), 6.80(\mathrm{~s}, 1 \mathrm{H}$, pyrazole $\mathrm{H}-4), 7.28-7.46(\mathrm{~m}$, $\left.10 \mathrm{H}, 2 \mathrm{C}_{6} \mathrm{H}_{5}\right), 8.32,8.44\left(2 \mathrm{~s}, 2 \mathrm{H}, \mathrm{D}_{2} \mathrm{O}\right.$-exchangeable, $\left.2 \mathrm{NH}\right)$. MS (relative intensity) m/z: 481. Analysis for $\mathrm{C}_{26} \mathrm{H}_{23} \mathrm{~N}_{7} \mathrm{OS}$ Calcd: C, 64.85; H, 4.81; N, 20.36; S, 6.66. Found: C, 64.62; H, $4.58 ; \mathrm{N}, 20.41 ; \mathrm{S}, 6.48 \%$.

\section{2-(3-Cyano-2-hydrazinyl-4,5,6,7-tetrahydrobenzo[b]thiophene)-4-cyano-3-oxo- phenyl- N-phenylpent-4-enamide (17)}

To a solution of compound 12a $(3.91 \mathrm{~g}, 0.01 \mathrm{~mol})$ in 1,4-dioxane (40 mL) containing piperidine $(0.50 \mathrm{~mL})$, benzaldehyde $(\mathbf{1 6}, 1.06 \mathrm{~g}, 0.01 \mathrm{~mol})$ was added. The reaction mixture was heated under reflux for $3 \mathrm{~h}$ then evaporated under vacuum. The remaining product was triturated with ethanol and the formed solid product was collected by filtration.

Compound 17: Yellow crystals from 1,4 dioxane, yield: $80 \%(3.836 \mathrm{~g})$; mp: $183-185^{\circ} \mathrm{C}$. IR $(\mathrm{KBr}): \mathrm{v} / \mathrm{cm}^{-1}=3523-3322(2 \mathrm{NH}), 3053\left(\mathrm{CH}\right.$-aromatic), $2877\left(\mathrm{CH}_{2}\right), 2227,2220(2 \mathrm{CN})$, 1718, 1684 (2 CO), $1636(\mathrm{C}=\mathrm{C}) .{ }^{1} \mathrm{H}$ NMR $\left(\mathrm{DMSO}_{\mathrm{d}}\right): \delta=1.67-1.74\left(\mathrm{~m}, 4 \mathrm{H}, 2 \mathrm{CH}_{2}\right), 2.13-$ 
$2.18\left(\mathrm{~m}, 4 \mathrm{H}, 2 \mathrm{CH}_{2}\right), 5.28(\mathrm{~s}, 1 \mathrm{H}, \mathrm{CH}=\mathrm{C}), 7.30-7.38\left(\mathrm{~m}, 10 \mathrm{H}, 2 \mathrm{C}_{6} \mathrm{H}_{5}\right), 8.31,8.42(2 \mathrm{~s}, 2 \mathrm{H}$, $\mathrm{D}_{2} \mathrm{O}$-exchangeable, 2NH). MS (relative intensity) $\mathrm{m} / \mathrm{z}$ : 479. Analysis for $\mathrm{C}_{27} \mathrm{H}_{21} \mathrm{~N}_{5} \mathrm{O}_{2} \mathrm{~S}$ Calcd: C, 67.62; H, 4.41; N, 14.60; S, 6.69. Found: C, 67.48; H, 4.53; N, 14.88; S, $6.43 \%$.

\section{2-(3-Cyano-2-hydrazinyl-4,5,6,7-tetrahydrobenzo[b]thiophene)-3-oxo-3-(2-oxo-2H- chromen-3-yl)-N-phenylpropanamide (19)}

To a solution of compound 12a $(3.91 \mathrm{~g}, 0.01 \mathrm{~mol})$ in 1,4-dioxane (40 mL) containing piperidine $(0.50 \mathrm{~mL})$, salicyladehyde $(1.22 \mathrm{~g}, 0.01 \mathrm{~mol})$ was added. The reaction mixture was heated under reflux for $5 \mathrm{~h}$ then evaporated under vacuum. The remaining product was triturated with ethanol and the formed solid product was collected by filtration.

Compound 19: Yellow crystals from 1,4 dioxane, yield: $80 \%$ (3.972 g); mp: 215-217 ${ }^{\circ} \mathrm{C}$. IR $(\mathrm{KBr}): \quad v / \mathrm{cm}^{-1}=3477-3342(2 \mathrm{NH}), 3055(\mathrm{CH}$-aromatic $), 2889\left(\mathrm{CH}_{2}\right), 2225(\mathrm{CN}), 1776$, 1734, 1667 (3 CO), $1638(\mathrm{C}=\mathrm{C}) .{ }^{1} \mathrm{H}$ NMR $\left(\mathrm{DMSO}-\mathrm{d}_{6}\right): \delta=1.69-1.73\left(\mathrm{~m}, 4 \mathrm{H}, 2 \mathrm{CH}_{2}\right), 2.21-$ $2.26\left(\mathrm{~m}, 4 \mathrm{H}, 2 \mathrm{CH}_{2}\right), 6.88(\mathrm{~s}, 1 \mathrm{H}$, coumarin $\mathrm{H}-4), 7.24-7.47\left(\mathrm{~m}, 9 \mathrm{H}, \mathrm{C}_{6} \mathrm{H}_{5}, \mathrm{C}_{6} \mathrm{H}_{4}\right), 8.33,8.44$ $\left(2 \mathrm{~s}, 2 \mathrm{H}, \mathrm{D}_{2} \mathrm{O}\right.$-exchangeable, 2NH). MS (relative intensity) $\mathrm{m} / \mathrm{z}$ : 496. Analysis for $\mathrm{C}_{27} \mathrm{H}_{20} \mathrm{~N}_{4} \mathrm{O}_{4} \mathrm{~S}$ Calcd: $\mathrm{C}, 65.31 ; \mathrm{H}, 4.06 ; \mathrm{N}, 11.28 ; \mathrm{S}, 6.46$. Found: $\mathrm{C}, 65.62 ; \mathrm{H}, 4.27 ; \mathrm{N}$, 10.99; S, $6.69 \%$.

\section{2-(3-Cyano-2-hydrazinyl-4,5,6,7-tetrahydrobenzo[b]thiophene)-4,4-dicyano-3-methyl-} $\mathrm{N}$-phenylbut-3-enamide (20) and 5-(2-Diazenyl-3cyano-4,5,6,7-tetrahydro benzo[b]thiophene)-2-amino-1,6-dihydro-4-methyl-6-oxo-1-phenylpyridine-3carbonitrile (21)

To a solution of compound 1 (3.66 g, $0.01 \mathrm{~mol})$ in DMF $(40 \mathrm{~mL})$ containing piperidine $(0.50$ $\mathrm{mL}$ ), malononitrile (3a, $0.66 \mathrm{~g}, 0.01 \mathrm{~mol})$ was added. The reaction mixture was heated under reflux for $3 \mathrm{~h}$ then left to cool and the formed solid product, upon pouring onto ice/water containing few drops of hydrochloric acid was collected by filtration. The solid showed two spots through TLC, the ethanol soluble product was identified to show product $\mathbf{2 0}$ while the ethanol insoluble was identified to give product $\mathbf{2 1}$.

Compound 20: Pale yellow crystals from ethanol, yield: $68 \%(2.818 \mathrm{~g})$; mp: $>290{ }^{\circ} \mathrm{C}$. IR $(\mathrm{KBr}): \mathrm{v} / \mathrm{cm}^{-1}=3449-3323(2 \mathrm{NH}), 3055\left(\mathrm{CH}\right.$-aromatic), 2955( $\left.\mathrm{CH}_{3}\right), 2227-2220(3 \mathrm{CN})$, $1693(\mathrm{CO}), 1633(\mathrm{C}=\mathrm{C}) .{ }^{1} \mathrm{H}$ NMR $\left(\mathrm{DMSO}_{-} \mathrm{d}_{6}\right): \delta=1.66-1.69\left(\mathrm{~m}, 4 \mathrm{H}, 2 \mathrm{CH}_{2}\right), 2.25-2.31(\mathrm{~m}$, $\left.4 \mathrm{H}, 2 \mathrm{CH}_{2}\right), 2.40\left(\mathrm{~s}, 3 \mathrm{H}, \mathrm{CH}_{3}\right), 7.29-7.40\left(\mathrm{~m}, 5 \mathrm{H}, \mathrm{C}_{6} \mathrm{H}_{5}\right), 8.42,9.29\left(2 \mathrm{~s}, 2 \mathrm{H}, \mathrm{D}_{2} \mathrm{O}-\right.$ exchangeable, $2 \mathrm{NH}$,). MS (relative intensity) $\mathrm{m} / \mathrm{z}$ : 414. Analysis for $\mathrm{C}_{22} \mathrm{H}_{18} \mathrm{~N}_{6} \mathrm{OS}$ Calcd: $\mathrm{C}$, 63.75; H, 4.38; N, 20.28; S, 7.74. Found: C, 63.92; H, 4.66; N, 20.32; S, $7.49 \%$.

Compound 21: Pale yellow crystals from DMF, yield: $79 \%(3.274 \mathrm{~g}) ; \mathrm{mp}$ : > $300{ }^{\circ} \mathrm{C}$. IR (KBr): v/cm ${ }^{-1}=3453-3343\left(\mathrm{NH}_{2}\right), 3053\left(\mathrm{CH}\right.$-aromatic), $2988\left(\mathrm{CH}_{3}\right), 2226,2220(2 \mathrm{CN})$, $1690(\mathrm{CO}), 1633(\mathrm{C}=\mathrm{C}) .{ }^{1} \mathrm{H}$ NMR $\left(\mathrm{DMSO}^{-} \mathrm{d}_{6}\right): \delta=1.64-1.69\left(\mathrm{~m}, 4 \mathrm{H}, 2 \mathrm{CH}_{2}\right), 2.18-2.26(\mathrm{~m}$, $\left.4 \mathrm{H}, 2 \mathrm{CH}_{2}\right), 2.73\left(\mathrm{~s}, 3 \mathrm{H}, \mathrm{CH}_{3}\right), 4.82\left(\mathrm{~s}, 2 \mathrm{H}, \mathrm{D}_{2} \mathrm{O}\right.$ exchangeable, $\left.\mathrm{NH}_{2}\right), 7.26-7.38(\mathrm{~m}, 5 \mathrm{H}$, $\mathrm{C}_{6} \mathrm{H}_{5}$ ), MS (relative intensity) m/z: 414. Analysis for $\mathrm{C}_{22} \mathrm{H}_{18} \mathrm{~N}_{6} \mathrm{OS}$ Calcd: C, 63.75; H, 4.38; N, 20.28; S, 7.74. Found: C, 64.02; H, 4.49; N, 20.11; S, $7.49 \%$

2-[2-(3-Cyano-4,5,6,7-tetrahydrobenzo[b]thiophen-2-yl)hydrazono]-3-(3,5-di- amino4H-pyrazol-4-ylidene)-N-phenylbutanamide (22a) and 3-(3-Amino-5-imino-1-phenyl$1 H$-pyrazol-4(5H)-ylidene)-2-[2-(3-cyano-4,5,6,7-tetrahydro-benzo[b]thiophen-2yl)hydrazono]-N-phenylbutanamide (22b)

To a solution of compound $20(2.07 \mathrm{~g}, 0.005 \mathrm{~mol})$ in ethanol $(40 \mathrm{~mL})$ either hydrazine hydrate $(\mathbf{8 a}, 0.30 \mathrm{~g}, 0.005 \mathrm{~mol})$ or phenyl hydrazine $(\mathbf{8 b}, 0.59 \mathrm{~g}, 0.005 \mathrm{~mol})$ was added. The reaction mixture was heated under reflux for $3 \mathrm{~h}$ then poured onto ice/water containing few drops of hydrochloric acid and the formed solid product was collected by filtration. 
Compound 22a: Pale yellow crystals from ethanol, yield: $68 \%(1.52 \mathrm{~g})$; mp: $290{ }^{\circ} \mathrm{C}$. IR $(\mathrm{KBr}): \mathrm{v} / \mathrm{cm}^{-1}=3462-3338\left(2 \mathrm{NH}_{2}, 2 \mathrm{NH}\right), 3058(\mathrm{CH}$-aromatic $), 2976\left(\mathrm{CH}_{3}\right), 2224(\mathrm{CN})$, $1688(\mathrm{CO}), 1638(\mathrm{C}=\mathrm{C}) .{ }^{1} \mathrm{H}$ NMR $\left(\mathrm{DMSO}^{\mathrm{d}} \mathrm{d}_{6}\right): \delta=1.62-1.65\left(\mathrm{~m}, 4 \mathrm{H}, 2 \mathrm{CH}_{2}\right), 2.25-2.32(\mathrm{~m}$, $\left.4 \mathrm{H}, 2 \mathrm{CH}_{2}\right), 2.84\left(\mathrm{~s}, 3 \mathrm{H}, \mathrm{CH}_{3}\right), 4.66,5.21\left(2 \mathrm{~s}, 4 \mathrm{H}, 2 \mathrm{NH}_{2}\right), 7.26-7.38\left(\mathrm{~m}, 5 \mathrm{H}, \mathrm{C}_{6} \mathrm{H}_{5}\right), 8.38$, $9.31\left(2 \mathrm{~s}, 2 \mathrm{H}, \mathrm{D}_{2} \mathrm{O}\right.$-exchangeable, $\left.2 \mathrm{NH}\right)$. MS (relative intensity) m/z: 446. Analysis for $\mathrm{C}_{22} \mathrm{H}_{22} \mathrm{~N}_{8} \mathrm{OS}$ Calcd: C, 59.18; H, 4.97; N, 25.09; S, 7.18. Found: C, 59.37; H, 4.73; N, 24.82; S, $7.28 \%$.

Compound 22b: Orange crystals from ethanol yield: $62 \%(1.62 \mathrm{~g}) ; \mathrm{mp}:>290{ }^{\circ} \mathrm{C}$. IR $(\mathrm{KBr})$ : $\mathrm{v} / \mathrm{cm}^{-1}=3460-3328\left(\mathrm{NH}_{2}, 3 \mathrm{NH}\right), 3054\left(\mathrm{CH}\right.$-aromatic), $2984\left(\mathrm{CH}_{3}\right), 2224(\mathrm{CN}), 1688(\mathrm{CO})$, $1636(\mathrm{C}=\mathrm{C}) .{ }^{1} \mathrm{H}$ NMR (DMSO-d $\left.\mathrm{d}_{6}\right): \delta=1.60-1.67\left(\mathrm{~m}, 4 \mathrm{H}, 2 \mathrm{CH}_{2}\right), 1.94-1.98\left(\mathrm{~m}, 4 \mathrm{H}, 2 \mathrm{CH}_{2}\right)$, $2.84\left(\mathrm{~s}, 3 \mathrm{H}, \mathrm{CH}_{3}\right), 4.68\left(\mathrm{~s}, 2 \mathrm{H}, \mathrm{D}_{2} \mathrm{O}\right.$ exchangeable, $\left.\mathrm{NH}_{2}\right), 7.23-7.36\left(\mathrm{~m}, 10 \mathrm{H}, 2 \mathrm{C}_{6} \mathrm{H}_{5}\right), 8.36$, 8.62, $9.33\left(3 \mathrm{~s}, 3 \mathrm{H}, \mathrm{D}_{2} \mathrm{O}\right.$-exchangeable, $\left.3 \mathrm{NH}\right)$. MS (relative intensity) $\mathrm{m} / \mathrm{z}: 522$. Analysis for $\mathrm{C}_{28} \mathrm{H}_{26} \mathrm{~N}_{8} \mathrm{OS}$ Calcd: C, 64.35; H, 5.01; N, 21.44; S, 6.14. Found: C, 64.29; H, 5.21; N, 21.73; S, $6.28 \%$.

\section{2-(3-Cyano-2-hydrazinyl-4,5,6,7-tetrahydrobenzo[b]thiophene)-3-(4,6-diamino-2 - thioxopyrimidin-5(2H)-ylidene)-N-phenylbutanamide (23a) and 2-(3-Cyano-2- hydrazinyl-4,5,6,7-tetrahydrobenzo $[b]$ thiophene)-3-(4,6-diamino-2-oxo pyrimidin- 5(2H)-ylidene)-N-phenylbutanamide (23b)}

To a suspension of compound $20(2.07 \mathrm{~g}, 0.005 \mathrm{~mol})$ in sodium ethoxide \{prepared by dissolving sodium metal $(0.46 \mathrm{~g}, 0.02 \mathrm{~mol})$ in absolute ethanol $(40 \mathrm{~mL})]$ either thiourea $(\mathbf{1 3 a}, 0.38 \mathrm{~g}, 0.005 \mathrm{~mol})$ or urea $(\mathbf{1 3 b}, 0.30 \mathrm{~g}, 0.005 \mathrm{~mol})$. The whole reaction mixture was heated in a boiling water bath for $3 \mathrm{~h}$ then poured onto ice/water containing few drops of hydrochloric acid (till $\mathrm{pH} 6$ ) and the formed solid product was collected by filtration.

Compound 23a: Yellow crystals from ethanol, yield: $58 \%(1.42 \mathrm{~g}) ; \mathrm{mp}$ : $>290^{\circ} \mathrm{C}$. IR (KBr): $\mathrm{v} / \mathrm{cm}^{-1}=3469-3312\left(2 \mathrm{NH}_{2}, 2 \mathrm{NH}\right), 3058(\mathrm{CH}$-aromatic $), 2978\left(\mathrm{CH}_{3}\right), 2222(\mathrm{CN}), 1684(\mathrm{CO})$, $1632(\mathrm{C}=\mathrm{C}) .{ }^{1} \mathrm{H}$ NMR $\left(\mathrm{DMSO}-\mathrm{d}_{6}\right): \delta=1.58-1.66\left(\mathrm{~m}, 4 \mathrm{H}, 2 \mathrm{CH}_{2}\right), 1.88-1.93\left(\mathrm{~m}, 4 \mathrm{H}, 2 \mathrm{CH}_{2}\right)$, $2.89\left(\mathrm{~s}, 3 \mathrm{H}, \mathrm{CH}_{3}\right), 4.69,5.44\left(2 \mathrm{~s}, 4 \mathrm{H}, 2 \mathrm{NH}_{2}\right), 7.23-7.38\left(\mathrm{~m}, 5 \mathrm{H}, \mathrm{C}_{6} \mathrm{H}_{5}\right), 8.22,9.33(2 \mathrm{~s}, 2 \mathrm{H}$, $\mathrm{D}_{2} \mathrm{O}$-exchangeable, 2NH). MS (relative intensity) m/z: 490. Analysis for $\mathrm{C}_{23} \mathrm{H}_{22} \mathrm{~N}_{8} \mathrm{OS}_{2}$ Calcd: C, 56.31; H, 4.52; N, 22.84; S, 13.07. Found: C, 56.07; H, 4.72; N, 22.63; S, 12.78 $\%$.

Compound 23b: White crystals from ethanol, yield: $73 \%(1.73 \mathrm{~g})$; mp:>290 ${ }^{\circ} \mathrm{C}$. IR $(\mathrm{KBr})$ : $\mathrm{v} / \mathrm{cm}^{-1}=3473-3348\left(2 \mathrm{NH}_{2}, 2 \mathrm{NH}\right), 3056(\mathrm{CH}$-aromatic $), 2966\left(\mathrm{CH}_{3}\right), 2220(\mathrm{CN}), 1690$, 1686 (2CO), $1635(\mathrm{C}=\mathrm{C}) .{ }^{1} \mathrm{H}$ NMR (DMSO-d $)$ ) $\delta=1.60-1.67\left(\mathrm{~m}, 4 \mathrm{H}, 2 \mathrm{CH}_{2}\right), 1.91-1.97(\mathrm{~m}$, $\left.4 \mathrm{H}, 2 \mathrm{CH}_{2}\right), 2.86\left(\mathrm{~s}, 3 \mathrm{H}, \mathrm{CH}_{3}\right), 4.68,5.40\left(2 \mathrm{~s}, 4 \mathrm{H}, 2 \mathrm{NH}_{2}\right), 7.25-7.40\left(\mathrm{~m}, 5 \mathrm{H}, \mathrm{C}_{6} \mathrm{H}_{5}\right), 8.28$, $9.38\left(2 \mathrm{~s}, 2 \mathrm{H}, \mathrm{D}_{2} \mathrm{O}\right.$-exchangeable, 2NH). MS (relative intensity) m/z: 474. Analysis for $\mathrm{C}_{23} \mathrm{H}_{22} \mathrm{~N}_{8} \mathrm{O}_{2} \mathrm{~S}$ Calcd: C, 58.21; H, 4.67; N, 23.61; S, 6.76. Found: C, 57.92; H, 4.81; N, 23.88; S, $7.03 \%$. 
$\mathrm{S}_{\mathrm{S}}^{\mathrm{NH}-\mathrm{N}=\mathrm{C}_{-\mathrm{CONHPh}}^{\prime}}$

1

2

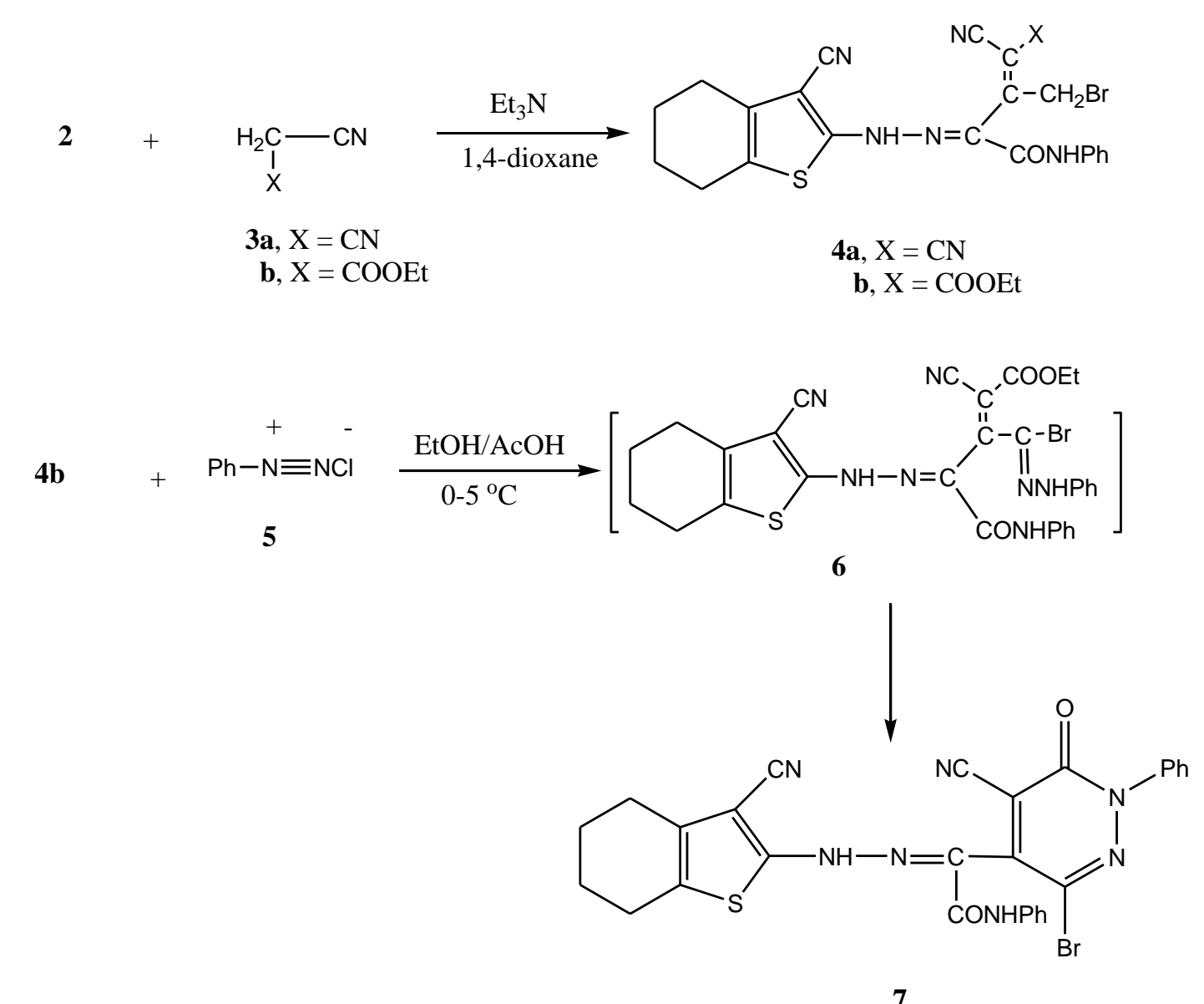

Scheme (1) 


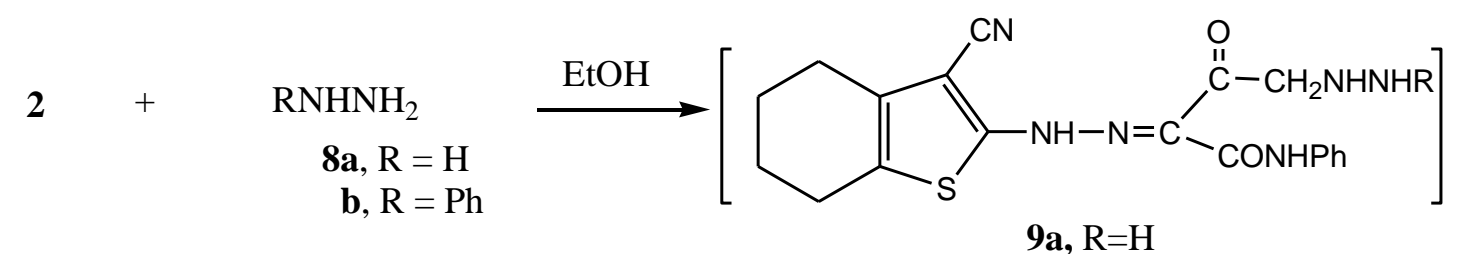

9a, $R=H$
b, $R=P h$

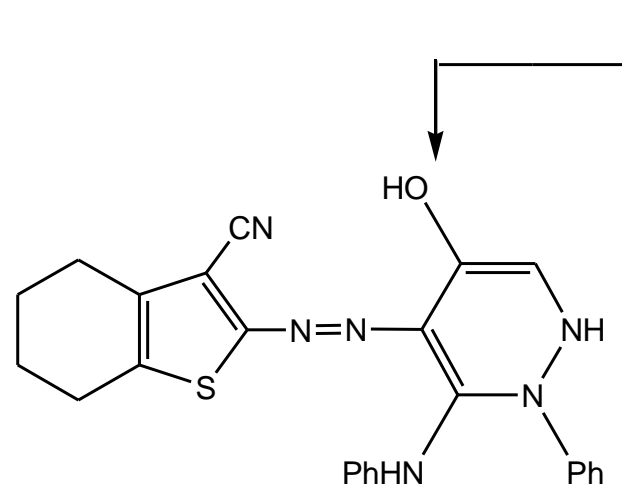

10b<smiles>N#Cc1c(NN=C(C(=O)CBr)C(=O)Nc2ccccc2)sc2c1CCCC2</smiles>

2

$$
\begin{array}{r}
\mathrm{KX} \\
\text { 11a, } X=\mathrm{CN} \\
\text { b, } X=\mathrm{SCN}
\end{array}
$$

$\mathrm{EtOH}$

$60{ }^{\circ} \mathrm{C}$<smiles>[X]CC(=O)C(=NNc1sc2c(c1C#N)CCCC2)C(=O)Oc1ccccc1</smiles>

$$
\begin{aligned}
\text { 12a, } X & =\mathrm{CN} \\
\text { b, } X & =\mathrm{SCN}
\end{aligned}
$$

Scheme (2) 


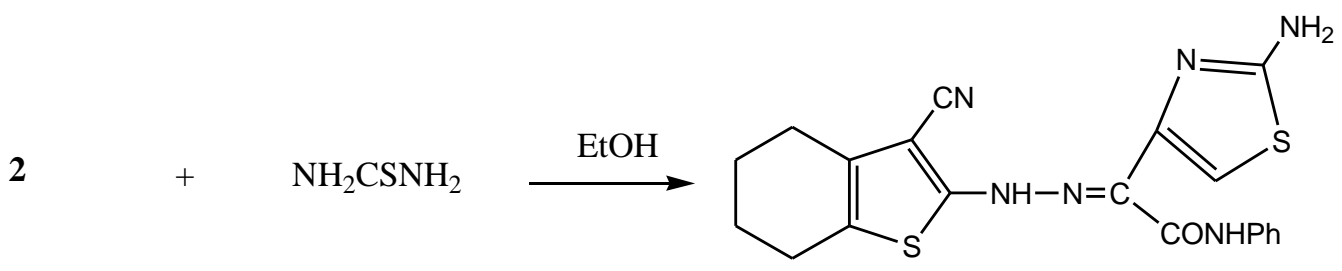<smiles>N#Cc1c(NN=C(C(=O)CCl)C(=O)Oc2ccccc2)sc2c1CCCC2</smiles>

$12 \mathrm{a}$
$\mathrm{RNHNH}_{2}$

8a, $\mathrm{R}=\mathrm{H}$

b, $\mathrm{R}=\mathrm{Ph}$<smiles>C=C(N)/C=C(\N)C(=NNc1sc2c(c1C#N)CCCC2)C(=O)c1ccccc1</smiles>

15a, $\mathrm{R}=\mathrm{H}$

b, $\mathrm{R}=\mathrm{Ph}$

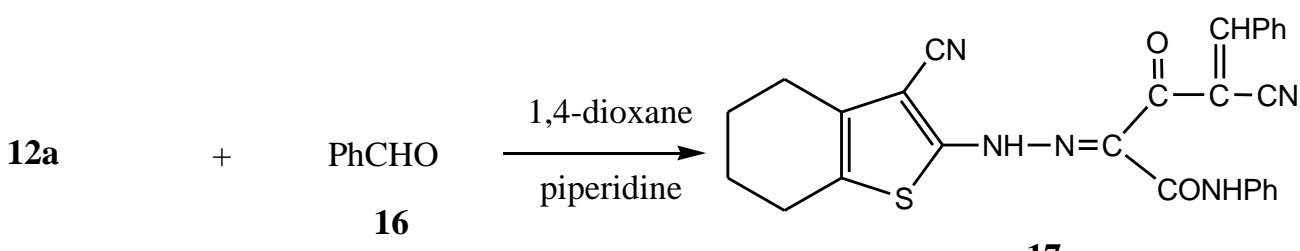

$12 \mathrm{a}+$<smiles>O=Cc1ccccc1O</smiles>

18 $\underset{\text { piperidine }}{\stackrel{\text { 1.4-dioxane }}{\longrightarrow}}$<smiles>N#Cc1c(N)sc2c1CCCC2</smiles>

Scheme (3) 


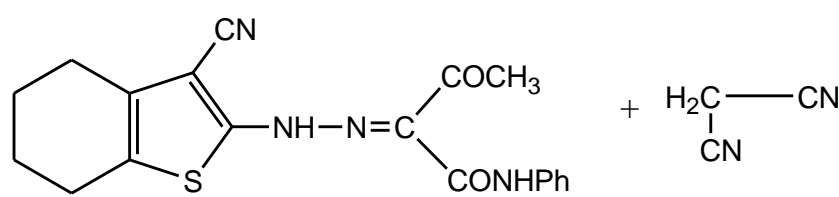

1

$3 a$

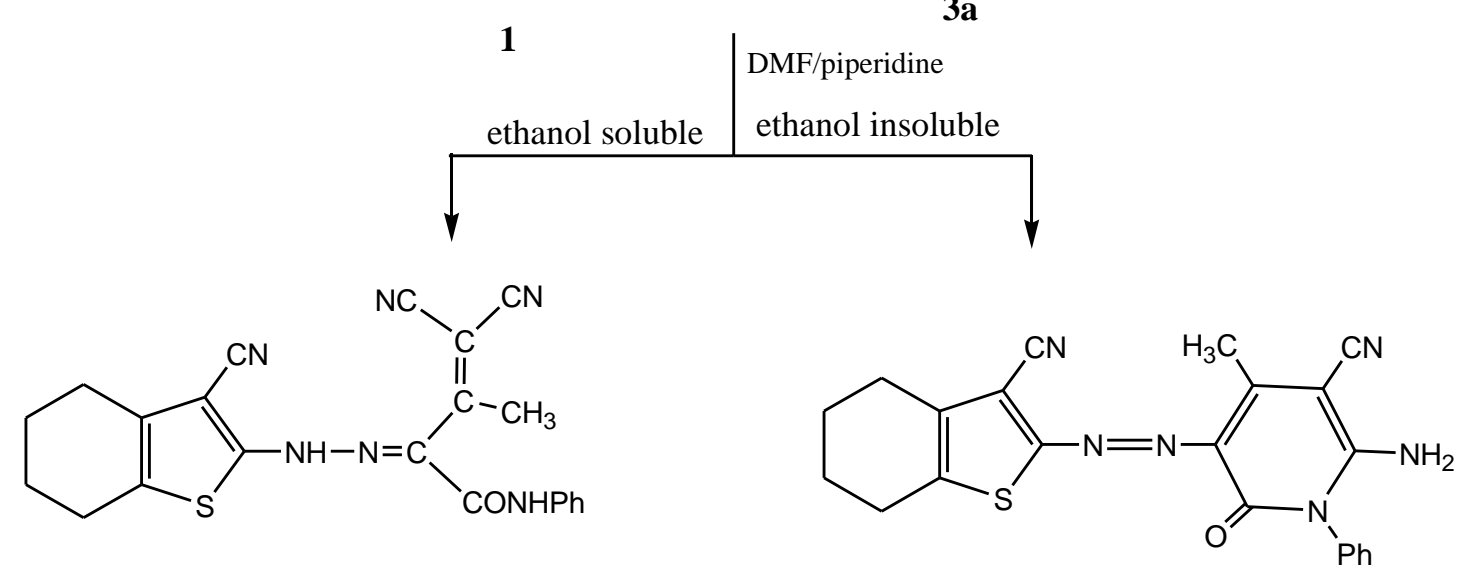

20

21

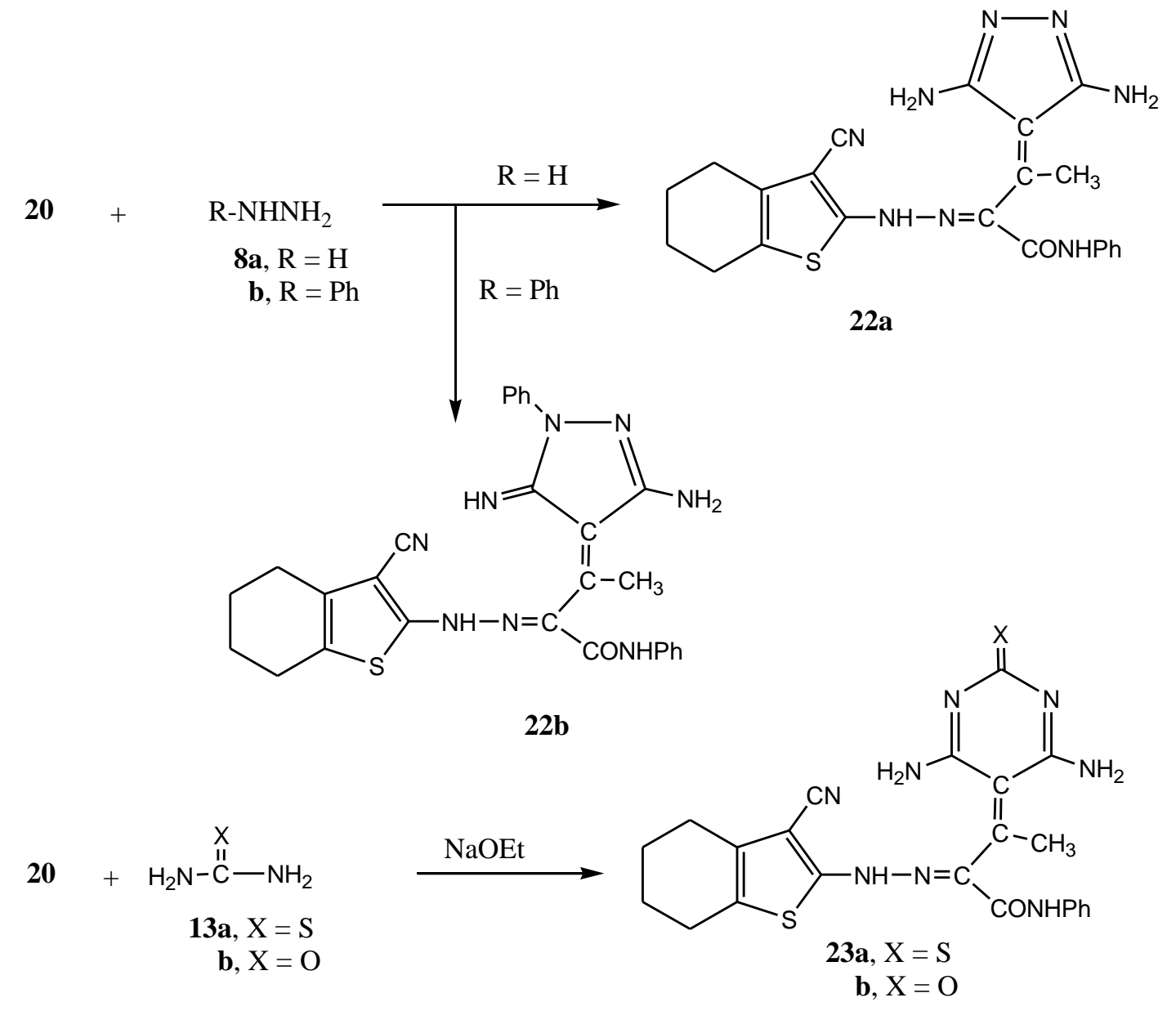

Scheme (4) 


\section{RESULTS AND DISCUSSION}

Recently, we were involved through comprehensive program involving the uses of 4,5,6,7-tetrahydrobenzo[ $b]$ thiophene derivatives (Mohareb et al., 2009) together with their further reactions with chemical reagents to give heterocyclic and fused heterocyclic derivatives with antitumor activities, In continuation of this program, we report here the reactivity of the 2-(3-Cyano-2-hydrazinyl-4,5,6,7-tetrahydrobenzo- [b]thiophene)-3-oxo-Nphenylbutanamide (1) with some chemical reagents. Thus, reaction of $\mathbf{1}$ with bromine in acetic acid solution to give the $\alpha$-bromocarbonyl compound $\mathbf{2}$. The structure of compound $\mathbf{2}$ was based on analytical and spectral data. Thus, the ${ }^{1} \mathrm{H}$ NMR spectrum showed two multiplets at $\delta 1.74-1.79 \& 2.11-2.18$ ppm indicating to the four $\mathrm{CH}_{2}$ groups, a singlet at $\delta$ $3.88 \mathrm{ppm}$ corresponding to the $\mathrm{CH}_{2}$ group, multiplet at $\delta 7.27-7.38 \mathrm{ppm}$ for the $\mathrm{C}_{6} \mathrm{H}_{5}$ group and two singlets, $\mathrm{D}_{2} \mathrm{O}$-exchangeable, at $\delta 8.32 \& 9.44 \mathrm{ppm}$ for the two $\mathrm{NH}$ groups. The reaction of compound $\mathbf{2}$ with either malononitrile (3a) or ethyl cyanoacetate $(\mathbf{3 b})$ in refluxing 1,4-dioxane containing a catalytic amount of triethylamine gave the condensate products $\mathbf{4 a}$ and $4 \mathbf{b}$ respectively. The structures of the latter products were based on analytical and spectral data. Thus, the ${ }^{1} \mathrm{H}$ NMR spectrum of $4 \mathbf{a}$ showed two multiplets at $\delta 1.71-1.76 \&$ 2.15-2.19 ppm indicating the four $\mathrm{CH}_{2}$ groups, a singlet at $\delta 3.93$ ppm corresponding to $\mathrm{CH}_{2}$ group, a multiplet at $\delta 7.28-7.39 \mathrm{ppm}$ for $\mathrm{C}_{6} \mathrm{H}_{5}$ group and two singlets, $\mathrm{D}_{2} \mathrm{O}$-exchangeable, at $\delta 8.39 \& 9.42 \mathrm{ppm}$ for the two $\mathrm{NH}$ groups. Moreover, the reaction of compound $\mathbf{4 b}$ with benzenediazonium chloride at $0-5{ }^{\circ} \mathrm{C}$ gave the pyridazine derivative 7 , its formation is explained in terms of the intermediate formation of the arylhydrazo derivative 6 (Scheme1).

The reaction of $\alpha$-oxobromo derivative $\mathbf{2}$ with either hydrazine hydrate (8a) or phenylhydrazine $(\mathbf{8 b})$ gave the pyridazine derivatives $\mathbf{1 0 a}$ and $\mathbf{1 0 b}$ respectively. Formation of the latter products was based on the intermediate formation $\alpha$-hydrazinoxo derivatives $9 \mathbf{a}$, b followed by water elimination. The structural elucidations were based on the obtained analytical and spectral data. Thus, the ${ }^{1}$ HNMR spectrum of 10a showed two multiplets at $\delta$ 1.73-1.78 \& 2.21-2.26 ppm indicating to the four $\mathrm{CH}_{2}$ groups, a singlet at $\delta 6.83 \mathrm{ppm}$ corresponding to the pyridazine $\mathrm{H}-3$ group, a multiplet at $\delta 7.29-7.42 \mathrm{ppm}$ for the $\mathrm{C}_{6} \mathrm{H}_{5}$ group, a singlet, $\mathrm{D}_{2} \mathrm{O}$-exchangeable at $\delta 8.28 \mathrm{ppm}$ for the $\mathrm{NH}$ group and a singlet at $\delta 9.38$ ppm for the $\mathrm{OH}$ group.

The reaction of compound 2 with either potassium cyanide (11a) or potassium thiocyanate (11b) gave either $\alpha$-oxonitrile derivative 12a or the $\alpha$-oxothiocyanate derivative 12b respectively (Scheme2).

On the other hand, the reaction of compound 2 with thiourea (13a) in refluxing ethanol gave the thiazole derivative 14. The analytical and spectral data were in agreement with the assigned structure. Next, we moved towards studying the reactivity of the $\alpha$ oxonitrile derivative 12a in order to form new heterocyclic compounds derivatives with potential biological activities. Thus, the reaction of 12a with either hydrazine hydrate (8a) or phenylhydrazine (8b) gave pyrazole derivatives $\mathbf{1 5 a}, \mathbf{b}$. On the other hand, the reaction of 12a with benzaldehyde (16) in refluxing 1,4-dioxane containing a catalytic amount of piperidine gave the benzal derivative 17. Moreover, the reaction of 12a with salicylaldehyde (18) gave the coumarin derivative 19 (Scheme3). The analytical and spectral data of the latter product were in agreement with the assigned structure.

The reaction of compound 1 with malononitrile (3a) in DMF/piperidine solution gave two isomeric products with the same molecular formula $\mathrm{C}_{22} \mathrm{H}_{18} \mathrm{~N}_{6} \mathrm{OS}$, the ethanol soluble product assigned the acyclic structure $\mathbf{2 0}$ while the ethanol insoluble product is the pyridine derivative 21. The structures of compounds $\mathbf{2 0}$ and $\mathbf{2 1}$ were based on analytical and spectral 
data. The dicyanomethino group present in compound $\mathbf{2 0}$ showed interesting reactivity towards the reaction with diamino reagents. Thus, the reaction of compound $\mathbf{2 0}$ reacted with either hydrazine hydrate (8a) or phenylhydrazine $\mathbf{( 8 b}$ ) gave the pyrazole derivatives $\mathbf{2 2 a}$ and $\mathbf{2 2 b}$ respectively. On the other hand the reaction of compound $\mathbf{2 0}$ with either thiourea (13a) or urea (13b) in sodium ethoxide solution to give the pyrimidine derivatives $\mathbf{2 3 a}$ and $\mathbf{2 3 b}$ respectively (Scheme4).

\section{Antitumor activity tests}

Reagents: Fetal bovine serum (FBS) and L-glutamine from Gibco Invitrogen Co. (Scotland, UK). RPMI-1640 medium from Cambrex (New Jersey, USA). Dimethyl sulfoxide (DMSO), doxorubicin, penicillin, streptomycin and sulforhodamine B (SRB) were from Sigma Chemical Co. (Saint Louis, USA).

Cell cultures: Three human tumor cell lines, MCF-7 (breast adenocarcinoma), NCI-H460 (non-small cell lung cancer), and SF-268 (CNS cancer) were used together with the normal cell lines the normal fibroblast cells (WI 38). MCF-7 was obtained from the European Collection of Cell Cultures (ECACC, Salisbury, UK) and NCI-H460 and SF-268 were kindly provided by the National Cancer Institute (NCI, Cairo, Egypt). They grow as monolayer and routinely maintained in RPMI-1640 medium supplemented with 5\% heat inactivated FBS, $2 \mathrm{mM}$ glutamine and antibiotics (penicillin $100 \mathrm{U} / \mathrm{mL}$, streptomycin 100 $\mu \mathrm{g} / \mathrm{mL}$ ), at $37^{\circ} \mathrm{C}$ in a humidified atmosphere containing $5 \% \mathrm{CO}_{2}$. Exponentially growing cells were obtained by plating $1.5 \times 10^{5}$ cells $/ \mathrm{mL}$ for MCF-7 and SF-268 and $0.75 \times 10^{4}$ cells $/ \mathrm{mL}$ for NCI-H460, followed by $24 \mathrm{~h}$ of incubation. The effect of the vehicle solvent (DMSO) on the growth of these cell lines was evaluated in all the experiments by exposing untreated control cells to the maximum concentration $(0.5 \%)$ of DMSO used in each assay.

Tumor cell growth assay: The effects of $\mathbf{2 - 2 3 a , b}$ on the in vitro growth of human tumor cell lines were evaluated according to the procedure adopted by the National Cancer Institute (NCI, USA) in the 'In vitro Anticancer Drug Discovery Screen' that uses the protein-binding dye sulforhodamine B to assess cell growth. Briefly, exponentially, cells growing in 96-wellplates were then exposed for $48 \mathrm{~h}$ to five serial concentrations of each compound (Skehan et al., 1990), starting from a maximum concentration of $150 \mu \mathrm{M}$. Following this exposure period adherent cells were fixed, washed, and stained. The bound stain was solubilized and the absorbance was measured at $492 \mathrm{~nm}$ in a plate reader (Bio-Tek Instruments Inc., Power wave XS, Wincoski, USA). For each test compound and cell line, a dose-response curve was obtained and the growth inhibition of $50 \%\left(\mathrm{GI}_{50}\right)$, corresponding to the concentration of the compounds that inhibited $50 \%$ of the net cell growth, was calculated as described elsewhere (Monks et al., 1991). Doxorubicin was used as a positive control and tested in the same manner. 
Table 1. Effect of compounds 2-23a,b on the growth of human tumor cell lines and a normal cell line

\begin{tabular}{|c|l|l|l|l|}
\hline \multirow{2}{*}{ Compound } & \multicolumn{4}{|c|}{$\left.\mathrm{GI}_{50}(\mu \mathrm{mol})^{-1}\right)$} \\
\cline { 2 - 5 } & MCF-7 & NCI-H460 & SF-268 & WI 38 \\
\hline $\mathbf{2}$ & $42.6 \pm 12.2$ & $36.6 \pm 8.6$ & $62.4 \pm 14.8$ & $22.3 \pm 6.0$ \\
\hline 4a & $32.4 \pm 10.6$ & $26.1 \pm 2.7$ & $28.9 \pm 6.8$ & $40.1 \pm 6.0$ \\
\hline 4b & $22.2 \pm 1.2$ & $32.6 \pm 1.4$ & $36.4 \pm 0.6$ & $32.1 \pm 4.8$ \\
\hline $\mathbf{7}$ & $14.6 \pm 2.4$ & $12.9 \pm 0.8$ & $11.8 \pm 0.6$ & $44.2 \pm 10.2$ \\
\hline $\mathbf{1 0 a}$ & $20.6 \pm 0.4$ & $24.3 \pm 0.8$ & $32 \pm 0.8$ & $4.2 \pm 1.8$ \\
\hline $\mathbf{1 0 b}$ & $38.4 \pm 1.8$ & $42.0 \pm 0.8$ & $22.5 \pm 1.1$ & $64.2 \pm 12.6$ \\
\hline $\mathbf{1 2 a}$ & $33.1 \pm 0.6$ & $27.3 \pm 1.4$ & $24.3 \pm 1.5$ & $62.5 \pm 22.6$ \\
\hline $\mathbf{1 2 b}$ & $0.6 \pm 0.2$ & $0.2 \pm 0.02$ & $0.2 \pm 0.05$ & $22.6 \pm 8.0$ \\
\hline $\mathbf{1 4}$ & $22.0 \pm 0.6$ & $28.0 \pm 0.4$ & $30.5 \pm 8.0$ & $56.2 \pm 12.9$ \\
\hline $\mathbf{1 5 a}$ & $33.9 \pm 17.5$ & $40.2 \pm 12.8$ & $52.0 \pm 9.0$ & $46.5 \pm 8.0$ \\
\hline $\mathbf{1 5 b}$ & $34.0 \pm 1.8$ & $46.0 \pm 0.8$ & $22.5 \pm 1.1$ & $12.3 \pm 2.6$ \\
\hline $\mathbf{1 7}$ & $0.01 \pm 0.004$ & $0.02 \pm 0.002$ & $0.01 \pm 0.001$ & $66.5 \pm 14.7$ \\
\hline $\mathbf{1 9}$ & $0.03 \pm 0.007$ & $0.02 \pm 0.008$ & $0.01 \pm 0.004$ & $>100$ \\
\hline $\mathbf{2 0}$ & $26.7 \pm 17.8$ & $24.2 \pm 12.6$ & $36.0 \pm 6.0$ & $72.1 \pm 22.3$ \\
\hline $\mathbf{2 1}$ & $28.7 \pm 11.5$ & $22.2 \pm 10$. & $22.0 \pm 8.0$ & $20.7 \pm 8.3$ \\
\hline $\mathbf{2 2 a}$ & $22.4 \pm 0.2$ & $22.6 \pm 1.4$ & $33.4 \pm 0.6$ & $40.3 \pm 10.6$ \\
\hline $\mathbf{2 2 b}$ & $10.2 \pm 0.4$ & $12.1 \pm 0.6$ & $18.3 \pm 0.5$ & $66.4 \pm 16.7$ \\
\hline $\mathbf{2 3 a}$ & $2.0 \pm 1.0$ & $3.6 \pm 1.4$ & $2.4 \pm 0.8$ & $70.4 \pm 22.6$ \\
\hline $\mathbf{2 3 b}$ & $20.0 \pm 0.6$ & $22.0 \pm 0.4$ & $31.5 \pm 8.0$ & $80.3 \pm 18.4$ \\
\hline Doxorubicin & $0.04 \pm 0.008$ & $0.09 \pm 0.007$ & $0.09 \pm 0.007$ & $>100$ \\
\hline & & & & \\
\hline
\end{tabular}

$\mathrm{GI}_{50}$ mean value \pm standard error of mean of 3 independent experiments performed in duplicate.

\section{Effect on the Growth of Human Tumor Cell Lines}

The effect of compounds 2-23a,b was evaluated on the in vitro growth of three human tumor cell lines representing different tumor types, namely, breast adenocarcinoma (MCF-7), non-small cell lung cancer (NCI-H460) and CNS cancer (SF-268) and the normal fibroblast cells (WI 38) after a continuous exposure for 48h. The results are summarized in Table 1. All of the tested compounds were able to inhibit the growth of the tested human tumor cell lines in a dose-dependent manner. The results indicated through Table 1 revealed that "compounds 17 and 19 showed the highest inhibitory effect against all the three tumor cell lines", such activity is higher than the reference doxorubicin.

While compounds $\mathbf{1 2 b}$ and 23a showed high inhibitory effects against non-small cell lung cancer (NCI-H460) and breast adenocarcinoma (MCF-7) and CNS cancer (SF-268) respectively, which are less than the reference doxorubicin. Compounds $\mathbf{2 , 4 a , 1 0 b , 1 2 a , 1 5 a}$ and 15b showed the lowest inhibitory effect. The rest of the compounds showed a moderate growth inhibitory effect. Comparing of 12a with 12b it is obvious that the presence of SCN group in 12b is responsible for the greater inhibitory effect towards the three cell lines than 
that of 12a. Similarly comparing compound $\mathbf{2 3 a}$ and $\mathbf{2 3 b}$, it is obvious that the presence of the sulpher atom in compound $\mathbf{2 3} \mathbf{a}$ is responsible for their reactivity over $\mathbf{2 3 b}$.

\section{ACKNOWLEDGMENT}

The authors would like to thank the research group working at both the National Research Center and the National Cancer Institute for recording the antitumor evaluation for the synthesized products. Moreover, the effort of Prof Dr. Amany El-Barairy, MSA University, Pharmacology Department, is greatly appreciated for her kind revisions for the pharmacological data.

\section{REFERENCES}

Eicher, T.; Hauptmann, S.; Speicher, A. (2003): The Chemistry of Heterocycles: Chapter 5, Five-Membered Heterocycles, Section 5.6 Thiophene; Wiley-VCH:New York, NY.

Foks, H., Pancehowska-Ksepko, D., Kedzia, A., Zwolska, Z., Janowiec, M., Augustvnowicz-Kopec, E. (2005): Syntheis and antibacterial activity of $1 H$ pyrazolo [3,4-b] pyrazine and -pyridine derivatives, II Farmaco., Volume, 60(6-7), Pages 513-517.

Gronowitz, S. (1991): The Chemistry of Heterocyclic Compounds: Thiophene and its Derivatives; Vol. 44, Part 4, Chapter 2. Wiley: New York, NY.

Kate, F.B.; Nicola, C.; Janet, D.C.; David, W.H.; Sandra, E.O.; Claire, A.M.; Richard, A.P.; Julie, A.T.; Jason, B.; Andrew, P.; Sian, R.; Judith, S.; Anna, L.V. and Andrew, P.T. (2004): Imidazo[1,2-b]pyridazines: a potent and selective class of cyclin-dependent kinase inhibitors, Bio. \& Med. Chem. Lett., Volume 14(9), Pages 2249-2252.

Kwan, P., Brodie, M.j. (2004): Phenobarbital for the treatment of epilepsy in the $21^{\text {st }}$ century: a critical review. Epilpesia, Volume 45(9), Pages 1141-1149.

Mohareb, R.M., Sherif, S.M. and Moustafa, H. E. (2009): The Reaction of Cyclohexan1,3-dione with Cyanomethylenes: Synthesis of Thiophenes and Their Fused Derivatives with Antifungal Activities, Phosphorous, Sulfur \& Silicon, Volume 184, Pages 2078-2096.

Monks, A.; Scudiero, D.; Skehan, P.; Shoemaker, R.; Paull, K.; Vistica, D.; Hose, C.; Langl, J.; Cronise, P.; Vaigro-Wolff, A.; Gray-Goodrich, M.; Campbell, H.; Mayo, J. and Boyd, M.J. (1991): Feasibility of a High-Flux Anticancer Drug Screen Using a Diverse Panel of Cultured Human Tumor Cell Lines, Natl. Cancer Inst. Volume 83, Pages 757-766.

Patil, A.D., Kumar, N.V., Kokke, W.C., Bean, M.F., Freyer, A.J., De Brosse, C., Mai, S., Truneh, A. and Carte, B. (1995): Novel Alkaloids from the Sponge Batzella sp.:Inhibitors of HIV gp120-Human CD4 Binding. J. Org. Chem., Volume 60(5), Pages 1182-1188.

Pawan, K.S. and Sawhney, S.N. (1997): Potent antiinflammatory 3-thiazole-4(5)-acetic acids of 1,2-benzisothiazole, Bio. \& Med. Chem. Lett., Volume 7, Pages 24272430 . 
Rahul, R. N.; Vijary, V. K.; Sachin, S. B. and Devanand, B. S. (2006): Synthesis of new series of 1-Aryl-1,4-dihydro-4-oxo-6-methyl-pyridazine-3-carboxylic acid as potential antibacterial agents, Eur. J. Med. Chem., Volume 40(12), Pages 13251330.

Skehan, P.; Storeng, R.; Scudiero, D.; Monks, A.; McMahon, J.; Vistica, D.; Warren, J.T.; Bokesch H.; Kenney, S. and Boyd, M. R. J. (1990): New Colorimetric Cytotoxicity Assay for Anticancer-Drug Screening, Natl. Cancer Inst. Volume. 82, Pages 1107-1112.

Snider, B.B., Chen, J., Patil, A.D., Freyer, A.J. (1996): Synthesis of the tricyclic portions of batzelladines A, B and D. Revision of the stereochemistry of batzel; ladines A and D, Tetrahedron Lett., Volume 37, Pages 6677-6680.

Tapia, R.A.; Alegria, L.; Pesson, D.C. and Cristian, S. (2003): Synthesis and antiprotozoal activity of naphthofuranquinones and naphthothiophene-quinones containing a fused thiazole ring, Bio. \& Med. Chem., Volume 11, Pages 2175-2182.

Wu, C.; Decker, E.R.; Blok, N.; Bui, H.; You, T.J.; Wang, J.; Bourgoyne, A.R.; Knowles, V.; Berens, K.L.; Holland, G.W.; Brock, T.A. and Dixon, R.A. F. (2004): Discovery, Modeling, and Human Pharmacokinetics of $N$-(2Acetyl- 4,6-dimethyl- phenyl)-3-(3,4-dimethyl- isoxazol-5-ylsulfamoyl)

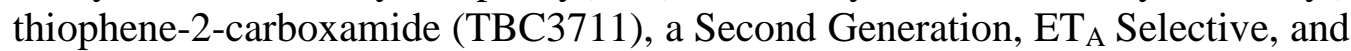
Orally Bioavailable Endothelin Antagonist, J. Med. Chem., Volume 47, Pages 1969-1986.

$$
\begin{aligned}
& \text { تطبيقات على 4,5,6,7 رباعى هيدروبنزو-ب-ثيوفين فى تخليق مشتقات البيريدازين والبيرازول }
\end{aligned}
$$

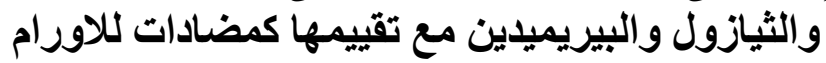

$$
\begin{aligned}
& \text { كرم احمد الثرقاوى } 1 \text { و فاتن اسماعيل حامد } 2
\end{aligned}
$$

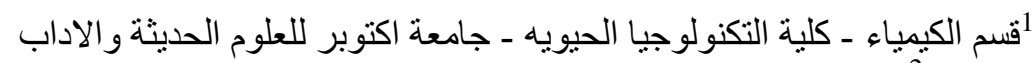

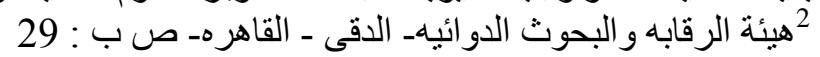

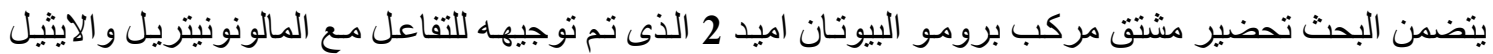

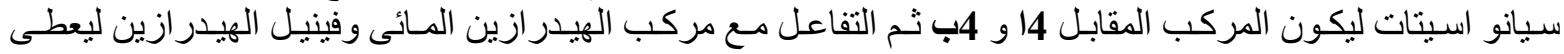

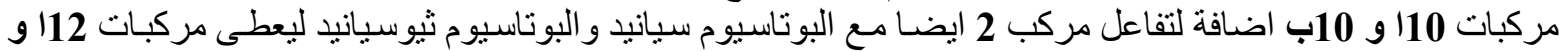

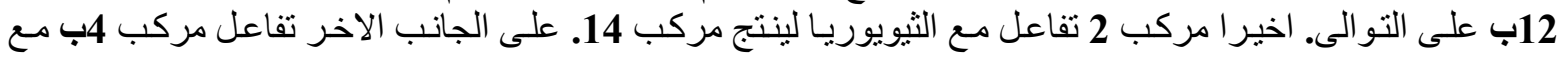

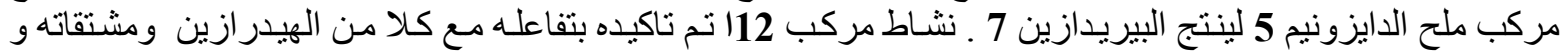

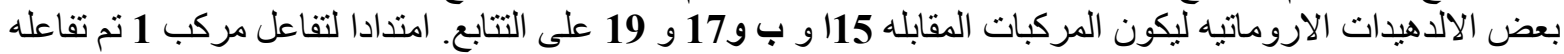

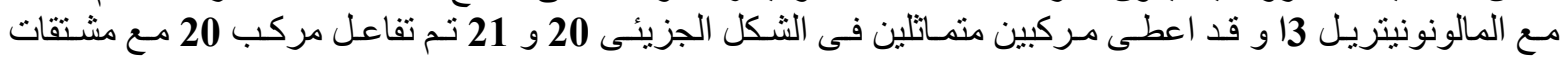

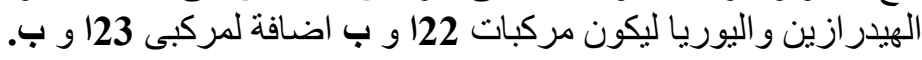

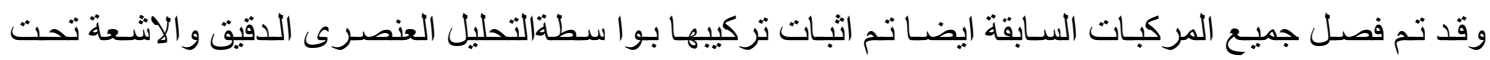
الحمر اء و الرنين النووى المغناطيسى ومطياف الكتله .

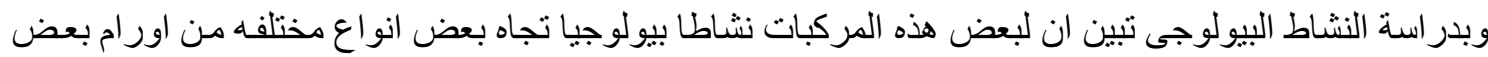

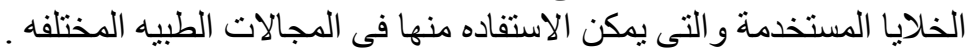

